45 (1) | 2016

En el río del Maíz (Madre de Dios)

Homenaje a Bernard Lelong

\title{
Máscaras sonoras y metamorfosis en el lenguaje ritual de los runas del Alto Pastaza (Amazonía, Perú)
}

Masques sonores et métamorphose dans le langage rituel des runa du Haut-

Pastaza (Amazonie, Pérou)

Sound Masks and Metamorphosis in Alto Pastaza Runa ritual Language

(Peruvian Amazon)

Andrea-Luz Gutiérrez Choquevilca

\section{OpenEdition}

Journals

Edición electrónica

URL: http://journals.openedition.org/bifea/7779

DOI: 10.4000/bifea.7779

ISSN: 2076-5827

Editor

Institut Français d'Études Andines

Edición impresa

Fecha de publicación: 1 abril 2016

Paginación: 17-37

ISSN: 0303-7495

Referencia electrónica

Andrea-Luz Gutiérrez Choquevilca, « Máscaras sonoras y metamorfosis en el lenguaje ritual de los

runas del Alto Pastaza (Amazonía, Perú) », Bulletin de l'Institut français d'études andines [En línea], 45

(1) | 2016, Publicado el 08 abril 2016, consultado el 05 noviembre 2020. URL : http://

journals.openedition.org/bifea/7779; DOI : https://doi.org/10.4000/bifea.7779

Les contenus du Bulletin de l'Institut français d'études andines sont mis à disposition selon les termes de la licence Creative Commons Attribution - Pas d'Utilisation Commerciale - Pas de Modification 4.0 International. 


\title{
Máscaras sonoras y metamorfosis en el lenguaje ritual de los runas del Alto Pastaza (Amazonía, Perú)*
}

\author{
Andrea-Luz Gutiérrez Choquevilca**
}

\begin{abstract}
Resumen
Basándose en el estudio pragmático de la tradición oral quechua del Alto Pastaza (Perú), el artículo examina el concepto de «máscara acústica» en el ámbito de la enunciación ritual y las prácticas de comunicación. Una atención dedicada a las técnicas narrativas de traducción (code-switching) empleadas en el mito de origen andoa-katsakati, como al proceso de transmisión de los cantos de cacería kayachina y de amor llakichina, revela que se le atribuye a la voz de ciertas entidades no humanas un poder de evocación central en las actividades rituales, tanto para el iniciado cazador como para los amantes quechuas. Se evidencia que la transformación de la voz mediante el uso de onomatopeyas específicas desempeña una función análoga a la de una «máscara», en el campo del sonido: es decir, el poder de mostrar la cara visible de los actores invisibles de las interacciones rituales, con fines de predación o de seducción. El estudio de la función performativa atribuida al sonido proporciona medios indispensables a la comprensión de la eficacia ritual quechua. Vemos que la «metamorfosis» del cazador o del amante está directamente vinculada con una manipulación del proceso de citación o mímesis de la voz de un locutor invisible o no humano. El análisis sugiere que las técnicas de comunicación acústicas observadas en el lenguaje ritual quechua juegan un papel clave no solo en la economía simbólica de las relaciones entre humanos y no humanos, sino también desde un punto de vista cognitivo y pragmático, en la transmisión de ciertas representaciones anímicas.
\end{abstract}

Palabras clave: Amazonía, quechua (Pastaza), máscara acústica, mito andoa-katsakati, cantos rituales, metamorfosis, iniciación, simbolismo sonoro, pragmática

* El trabajo de campo realizado para el estudio de la comunicación ritual de los runa amazónicos ha recibido el apoyo del Instituto Francés de Estudios Andinos (IFEA), del Musée du quai Branly (París) y del Centre national de la recherche scientifique (CNRS/EREA-Legs Lelong). Quisiéramos agradecer en particular a Jean-Pierre Chaumeil, Bonnie Chaumeil, Valentina Vapnarsky y Aurore Monod-Becquelin, primero por su amistad y segundo por haber aceptado compartir a lo largo de este trabajo de análisis sus reflexiones críticas e inspiraciones; también agradezco a Jean-Joinville Vacher y Henry Godard, exdirectores del IFEA, por su apoyo incondicional durante mis estadías en el campo.

** École Pratique des Hautes Études (EPHE), Laboratoire d'Anthropologie Sociale UMR 7130 Collège de France / LESC-UPO. E-mail: Andrea-Luz.Gutierrez-Choquevilca@ephe.sorbonne.fr 


\title{
Masques sonores et métamorphose dans le langage rituel des runa du Haut-Pastaza (Amazonie, Pérou)
}

\section{Résumé}

Cet article se fonde sur l'étude pragmatique de la tradition orale quechua du Haut-Pastaza. II examine le concept de «masque acoustique » dans le cadre de l'énonciation rituelle et des pratiques de communication. L'accent mis sur les techniques narratives de traduction (code-switching) utilisées dans le mythe d'origine andoa-katsakati, et sur le processus de transmission des chants de chasse kayachina et d'amour llakichina, révèle que l'on attribue à la voix de certaines entités non-humaines un pouvoir central d'évocation dans les activités rituelles, aussi bien pour le chasseur initié que pour les amants quechua. Il devient évident que la transformation de la voix à travers les onomatopées spécifiques joue, dans le registre sonore, une fonction analogue à celle d'un « masque » : c'est-à-dire le pouvoir de montrer la face visible des acteurs invisibles des interactions rituelles, à des fins de prédation ou de séduction. L'étude de la fonction performative attribuée au son fournit les moyens indispensables à la compréhension de l'efficacité rituelle quechua. Ainsi, la « métamorphose » du chasseur ou de l'amant est directement reliée à une manipulation du processus de citation ou mimesis de la voix du locuteur invisible ou non-humain. L'analyse suggère que les techniques de communication acoustiques observées dans le langage rituel quechua jouent un rôle-clé, non seulement dans l'economie symbolique des relations entre humains et non-humains, mais aussi du point de vue cognitif et pragmatique, dans la transmission de certaines représentations animistes.

Mots-clés : Amazonie, quechua (Pastaza), masque acoustique, mythe andoa-katsakati, chants rituels, métamorphose, initiation, symbolisme sonore, pragmatique

\section{Sound Masks and Metamorphosis in Alto Pastaza Runa ritual Language (Peruvian Amazon)}

\begin{abstract}
Based on a study of oral Quechua tradition in the Alto Pastaza (Peru), this article examines the concept of «acoustic masks» in the context of ritual enunciation and communication practices. A focus on code-switching techniques used in the andoa-katsaki origin myth, as well as on the transmission process of kayachina hunting songs and llakichina love songs, reveals that the voices of certain nonhuman entities are invested with power, which is central to ritual activities for the initiated hunter as well as for Quechua lovers. It becomes evident that voice transformation through the use of specific onomatopoeias holds a role similar to that of a «mask», in the field of sound: that is, the power to show the visible side of the invisible actors of ritual interaction, directed towards predation or seduction. The study of the performative role attributed to sound offers indispensable tools in order to understand the effectiveness of Quechua ritual. «Metamorphosis» of the hunter or the lover is directly linked to a manipulation of the process of citation or mimesis of the voice of an invisible or non-human speaker. This analysis suggests that the acoustical communication techniques observed in Quechua ritual language play a key role not only in the symbolic economy of the relationships between humans and non-humans, but also from a cognitive and pragmatic point of view in the transmission of certain animistic representations.
\end{abstract}

Keywords: Amazon, (Pastaza) Quechua, acoustic mask, andoa-katsakati myth, ritual songs, metamorphosis, initiation, sound symbolism, pragmatics 


\section{INTRODUCCIÓN}

«Ñukaka tukurisha: ketereere! nishka».

Yo me transformaré: «ime he vuelto cadáver!» dijo.

El tema central de la metamorfosis estimuló fructuosas reflexiones en el americanismo tropical. Sin embargo, el énfasis sobre la dimensión visual — piel, ropa, imágenes chamánicas, ornamentos corporales y máscaras rituales—, como matriz de proyección y transformación del sujeto ritual ha dejado la dimensión sonora menos analizada. Contamos con honrosas excepciones (Seeger, 1981; 1987; Hill, 1993; Basso, 1985; Olsen, 1996; Beaudet, 1997 [1977]; Menezes Bastos, 1990; Piedade, 1997; Mello, 2005; Fausto et al., 2011). Los rituales de las flautas sagradas proporcionan un caso digno de interés, ya que la eficacia del ritual de iniciación masculina radica precisamente en una asimetría radical entre la modalidad perceptiva visual y la auditiva1. Tanto en el yurupari de los grupos tukano del noreste amazónico como en otros rituales xinguanos, el sonido de las flautas revela un secreto a la vez disimulado a la vista — prohibido a las mujeres y declarado en su propia dimensión sonora (Montagnani, 2011; Hill \& Chaumeil, 2011). Sin embargo, en las regiones amazónicas donde no existe el complejo ritual de las flautas sagradas, la agentividad propia del sonido en la comunicación ritual ha sido menos analizada. En el campo del chamanismo, un cierto desinterés en la comunicación paraverbal y sonora podría estar vinculada con la idea común de que la comunicación ritual está caracterizada por una opacidad semántica constitutiva 2 . Es emblemática la observación de Graham Townsley (1993) a propósito de la palabra yaminahua usada para designar los cantos terapéuticos koshuiti: tsai yoshto yoshto «lengua torcida», refiriéndose a un lenguaje especial, donde abundan metáforas enigmáticas, sintagmas arcaicos y alteraciones fonológicas del lenguaje ordinario3. Sin embargo, aunque la observación yaminahua sin duda es empíricamente válida en la mayoría de las etnografías amazónicas, quisiéramos matizar en este artículo el carácter de opacidad de la

1 Una gran parte de la eficacia del ritual está basada en la percepción auditiva ya que la visión de los instrumentos está totalmente prohibida para las mujeres y los no iniciados (Véanse Hill \& Chaumeil, 2011; Goulard \& Karadimas, 2011).

2 Se refiere a la observación de que la mayoría de los cantos enunciados por los chamanes son casi incomprensibles por los no iniciados. Véanse también Overing, 1990; Olsen, 1996: 157-159; Déléage, 2009; Severi, 2007. La propuesta hecha por parte de Claude Lévi-Strauss (1949) acerca de la eficacia simbólica del lenguaje ritual como «mise en scène» de las estructuras mítico-sociales encontró dificultades tanto empíricas como analíticas. Se ha revelado en varios trabajos que la dimensión semántica está a menudo, para no decir siempre, subordinada a la lógica pragmática de las interacciones rituales (Houseman, 2008).

3 Según el autor, esa «opacidad cognitiva» primitiva del lenguaje ritual amerindio podría justificarse por la ambigüedad irreductible del referente del discurso (Townsley, 1993: 465): «Yoshi [spirits] are real beings who are both 'like and not like' the things they animate. They have no stable and unitary nature and thus, paradoxically the 'seeing as' of 'twisted language' is the only way of adequately describing them. Metaphor here is not inproper naming but the only proper naming possible». Otro argumento sería la observación de que el especialista ritual es el único ser capaz de comunicar con los espíritus y de adoptar, digamos, «sin riesgo» una «perspectiva» no humana. 
palabra ritual, enfocándonos en la función pragmática de ostensión proporcionada por el sonido en el momento hic et nunc de las interacciones rituales. Se sugiere a partir de la etnografía de los runas 4 _ grupos quechuahablantes del Alto Pastaza en la Amazonía peruana-, que la voz del enunciador ritual contribuye a esbozar un paisaje sonoro inmediatamente perceptible por los no iniciados, contribuyendo así a la atenuación de la asimetría cognitiva entre el maestro cantando y los novicios, entre la entidades visibles y los actores invisibles del teatro ritual. Donde la visión es o bien prohibida - en el caso de los rituales tipo Yurupari- o bien imposible, el poder performativo del sonido en su forma ritual se expresa a menudo a través de la convergencia entre el acto de nombrar y actualizar de una manera ostensiva la presencia - la misma voz- de las entidades convocadas en el espacio ritual (Olsen, 1996; Hill, 1993; Gutiérrez Choquevilca, 2011). Lo audible se vuelve visible a los iniciados, a la vez como imagen mental y como máscara efémera de las entidades invisibles.

Basándonos en el estudio pragmático de la tradición oral de los runas, sugerimos examinar la opacidad del discurso ritual desde otra perspectiva, tomando en consideración que la materia sonora del discurso ritual no solo juega un papel clave como mnemotécnica indígena sino también como instrumento evocador fundamental, estimulando y transformando las interacciones entre los protagonistas rituales, visibles e invisibles, humanos o no humanos.

A partir de la hipótesis de que el sonido proporciona a los auditores del ritual una percepción sensible de la cara auditiva de los invisibles, examinaremos en este artículo la pertinencia del concepto de «máscara sonora» en el ámbito de la enunciación ritual. Se evidencia a través de la tradición oral de los runas, que la transformación de la voz mediante el uso de onomatopeyas, gritos, silbidos, desempeña una función análoga a la de una «máscara», en el campo del sonido: es decir, el poder de mostrar la cara visible de los actores invisibles de las interacciones rituales ${ }^{5}$. El estudio de la representación indígena de la voz y de la función performativa atribuida al sonido como «máscara» ocultando la identidad humana del locutor nos proporciona medios indispensables para la comprensión de la eficacia ritual de los cantos kayachina y llakichina de los runas amazónicos. Veremos que dicha «transformación» del locutor está directamente vinculada con una manipulación del proceso de citación o mímesis de la voz de una entidad invisible o no humana. El análisis sugiere que las técnicas de comunicación sonoras observadas en el lenguaje ritual quechua juegan un papel clave no solo en la economía simbólica de las relaciones entre humanos y no humanos, sino

4 Los grupos quechua de la Amazonía peruana se autodenominan runa o inga y se ubican en la zona de los ríos Pastaza, Capahuari y Siwin; otras comunidades están ubicadas en los orillas del río NapoCuraray, Tigre y en la zona de Lamas en la parte peruana. La etnogénesis de los quichua amazónicos se ha desarrollado durante la época colonial, a partir del siglo XVI, mediante la difusión del quechua como lingua franca por los misioneros en las tierras bajas peruanas y ecuatorianas.

5 Los estudios que se dieron sobre el tema de los ornamentos visuales hacen resaltar que las máscaras tienen un valor menos icónico que indexical: no «representan» entidades invisibles en el estricto sentido, demuestran y revelan la presencia efectiva de las entidades involucradas en el marco ritual (Fausto, 2011; Goulard \& Karadimas, 2011). 
también desde un punto de vista cognitivo y pragmático, en la transmisión de ciertas representaciones anímicas.

\section{MUERTE, MUDA Y HETEROGLOSIA}

Al llegar por primera vez a la comunidad de Capahuari en el mes de febrero de 2004, en la ribera oriental del alto río Pastaza en la región noroeste del Oriente peruano (provincia de Datem del Marañón, Loreto), dudaba encontrar todavía huellas de los últimos descendientes de los «andoanos» de la familia Zapara. Esta estrategia de camuflaje étnico de los miembros zaparo entre los quechua amazónicos ha sido mencionada en la literatura (Reeve, 1993-1994: 21; Taylor, 2007: 155). Encontré en la comunidad Capahuari a los que se autodenominan runa — «gente» en quechua_, es decir tres ayllu mayoritarios — grupos de parentescopracticando el intercambio matrimonial: principalmente los cariahanu, mucushua y arawanaza6. A pesar del carácter obviamente pluriétnico de la zona, fue una sorpresa constatar la actitud de literal amnesia que los individuos exhibían acerca de sus raíces étnicas cuasiextintas: los andoa-katsakati. A menudo, la confesión sobre la posibilidad de una filiación con el grupo zaparo se limitaba a este punto de humor. Los jóvenes mushu exclamaban:

«Katsakati Runakunaka kawsapayarka kayachipayashkakuna: Kararaha Supay! Ka'ka' ra' ha'! Shamuhushpa kayka uyaripayanahun atalpashina!»: «Los katsakati eran los que Ilamaban al demonio Kakaraha Supay! iCuando llegaba Kakaraha, se oía de lejos la voz de una pájaro similar a la gallina!».

Al escuchar «Ka'ka'ra'ha'!», el sonónimo del demonio Supay temido por los antepasados, los jóvenes se echaban a reír a carcajadas... Mediante el grito y con cierta ironía, se hacía hincapié en sus orígenes andoa-katsakati (Gutiérrez Choquevilca, 2012: 54-64).

Entre los runa, el poder originario de metamorfosis atribuido a la voz se narra en un fragmento de mito de origen famoso, cuya peculiaridad es el uso de la heteroglosia. La transformación animal de los ancestros nawpa runa, antiguos miembros del grupo andoa o shimigaye hoy en día desaparecidos, está vinculada con el acto de proferir palabras sencillas del idioma extinto «katsakati» perteneciendo a la familia lingüística zaparoana7: «pekereere naana nasekeree», que significarían, según los recuerdos del narrador y los datos lingüísticos recogidos por Peeke (1954): «Estoy cambiando de piel» o también «Me estoy muriendo». En el mito, aludiendo a la

6 Sin contar con los intercambios matrimoniales frecuentes con los miembros de los grupos jivaroachuars vecinos de Titiyacu, anakman (hacia arriba) o Siwin, urayman (hacia abajo). Varios individuos me confiaron sus raíces sarayaku, pues eran originarios del Alto Pastaza ecuatoriano, en particular los que llevaban el apellido (kasta en quechua) Sandi y Dahua. La demografía del pueblo identificado como «Quechua del Pastaza» no ha dejado de crecer en los últimos años alcanzando los 6000 individuos dispersos en 23 comunidades en una distancia de $250 \mathrm{~km}$ en las orillas del río Pastaza.

7 El idioma katsakati identificado como shimigaye estaría emparentado con el idioma andoa de la familia lingüística zaparo (Peeke, 1954; Peeke \& Sargent, 1953; Michael et al., 2009). 
desaparición de los ancestros andoa, se representa a la muerte como una forma refinada de muda lingüística.

\author{
[...] «Kwintashu! \\ nishkashi apuka Suruka. \\ Akuychiy kawsashunchina! ñukanchika \\ mana kashunchu kay runa \\ shuk tukunka churu, \\ shuk tukunka kutu \\ shuk tukunka chuwa \\ shuk tukunka kukumpi, chilik, taruna: \\ chaykuna tukushunchina!» \\ nishkashi.
}

\section{Kunkumpi nishka: \\ «Ñukaka tukurisha: \\ ketereere! \\ nishka. Rimashka chasna i tukuy chaykuna munashkakuna: \\ «ketereere \\ ketsono'párti \\ ketsono'párti \\ pekeno'párti \\ naana nasekeree \\ pekereere \\ naana nasekeree».}

Chaytashi rimashkashi chay kunkumpika:

«iÑuka tukurisha!»

nishkashi. [...]
«iHablemos en asamblea!»

Dijo el jefe Sarigueya.

«iVivimos ahora!

Dejemos de ser estos humanos, sino que uno se transformará en mono chuwa

(Lagotrix sp.),

otro se transformará en mono kutu

(Alouatta seniculus),

otro se transformará en mono chuwa

(Ateles paniscus), otro se transformará en caracol kukumpi, otro en grillo, serpiente taruna: iHay que metamorfosearnos ahora mismo!», dice que dijo.

Caracol kukumpi dijo:

«Yo me transformaré:

[ime he vuelto cadáver!]»

dijo. Empezó a pronunciar estas palabras y

todos querían transformarse :

«[ime he vuelto cadáver!]

[el que se muere]

[el que se muere]

[el que se malogra]

[así dicen los ancianos]

[iestoy cambiando de piel!]

[así dicen los ancianos]»

Pronunciando estas palabras el caracol

kukumpi dijo:

«iYo me terminaré (moriré)!» [...]

El acto de proferir palabras ininteligibles por los runas actuales dejó a los ancestros transformados en caracoles, grillos y serpientes, es decir en seres reconocidos por su facultad de muda y metamorfosis, de muerte y renacimiento. En este mito, se resalta la homología entre la desaparición histórica de los ancestros shimigaye, dando paso a la etnogénesis de los runas actuales, y la emergencia de las discontinuidades entre las especies naturales: respeto a la comunicación ordinaria, ambos procesos pueden ser interpretados como la consecuencia de una alteración íntima de la voz humana, una heteroglosia originaria. Se destaca una definición pragmática de la metamorfosis animal y étnica, vinculada en ambos casos con la adopción de un idioma ajeno o no humano, fenómeno que cabe considerar como extendido en la Amazonía (Seeger, 1987: 61; Basso, 1985). La adopción de la técnica narrativa del code-switching en el discurso mitológico no solo proporciona al público receptor del mito (principalmente formado por una pequeña asamblea de jóvenes y niños) el ejemplo de una metamorfosis 
posible de la voz del narrador — mediante el uso de una entonación y un patrón de acentuación extraños-, pero también les impone una lectura muy diferente del régimen de la temporalidad «histórica» surgida del contacto con las misiones dominicanas o jesuitas. En este caso y a diferencia de lo que menciona la Etnografía acerca de la representación indígena del proceso civilizador transformando a los infieles awka («salvaje») en «buenos cristianos» alli runas, la tripartición del tiempo en los periodos incluyendo el «tiempo de los antepasados» unay, el «tiempo del inicio o de los abuelos» callari uras y el tiempo actual kunan pacha, no parece relevante para entender la desaparición del grupo andoa shimigaye. Recordamos la palabra de Kukumpi en el mito: «ketereere ime he vuelto cadáver!». En vez de una oposición «salvaje»-«civilizado», la muerte o metamorfosis de la etnia shimigaye estaría asimilada a una forma críptica de traducción, code-switching entre el idioma quechua y katsakati. En este sentido, si bien la mayoría de los runas actuales no hablan ni entienden el idioma katsakati — ahora declarado extinto por las autoridades peruanas - la palabra mítica proporciona el marco institucional y las condiciones ideales para la estabilización de un vocabulario mínimo. Este léxico está transmitido mediante una proposición reflexiva paradójica «iMe he vuelto cadáver! (...) / El que se muere / Así dicen los ancianos» (ketereere / ketsonópárti / naana nasekeree) que toma como objeto de transmisión la representación de su propia desaparición, su aniquilación como lengua o código semiótico compartido. Según esta interpretación mito-histórica, el proceso de cambio cultural podría coincidir con un doble proceso, fisiológico (muda y/o «muerte», es decir al menos «transformación») y semiótico (un dispositivo de traducción lingüística).

\section{PERMUTACIONES SEMIÓTICAS Y RELACIONES INTERESPECÍFICAS}

¿Cuáles son las otras formas de traducción intersemióticas conocidas en las interacciones concretas y ordinarias que los runas establecen con los seres de su entorno próximo? Más allá de lo que sugiere el mito, conviene resaltar que la transformación y la manipulación de los sonidos por la voz humana prevalece en las prácticas de subsistencia más cotidianas de los runas, especialmente en el marco de las actividades cinegéticas designadas por el eufemismo chawpita maskakri- «ir a buscar en el centro» o puri- «caminar». Las principales técnicas de caza incluyen el uso de la cerbatana pukuna con el veneno ampi, de las trampas tuklla y a menudo de la escopeta illapa. Disparar sin falla requiere una estrategia eficaz de imitación sonora de la voz animal, una técnica llamada por la nominalización de la raíz verbal katichi-, refiriéndose literalmente al acto de «hacer seguir o hacer responder» a la presa9. Las Ilamadas varían según el interlocutor y

8 Véanse Whitten, 1976: 46-51; Reeve, 1988: 92; 1993-1994; Muratorio, 1987. A nivel regional, difiere también de la descripción de la experiencia del cambio cultural como un proceso patogénico entre los vecinos achuars (Taylor, 2007: 154).

9 Las señales se dirigen al mono araña chuwa (Ateles paniscus), al mono nocturno sipuru (Pithecia monachus) o hacia los pájaros como el pawshi (Mitu salvini), el yami (Psophia crepitans), el karuntsi (Penelope jacquacu) o el tucán sikwanka (Ramphastos sp.). 
el mensaje: su intensidad, su frecuencia y el ritmo. Pueden estar acompañadas de gestos, como el gesto de golpear nueces para hacer sentir la presencia de frutos al mono machin (Cebus, sp.). Existen varias técnicas de silbido: la expiración del aire a través de una hoja vibrando entre los dos dedos (fig. 1), la técnica del silbido bilabial o la vibración de los labios apresurados contra la espalda de la mano derecha -el aire es inspirado- - El movimiento de la mano hacia el exterior permite producir variaciones de intensidad. Cabe mencionar que similares técnicas de cacería están difundidas entre la mayoría de los grupos amazónicos y han sido mencionadas en Brasil, entre los wayãpis (Tupis) (Beaudet, 1997

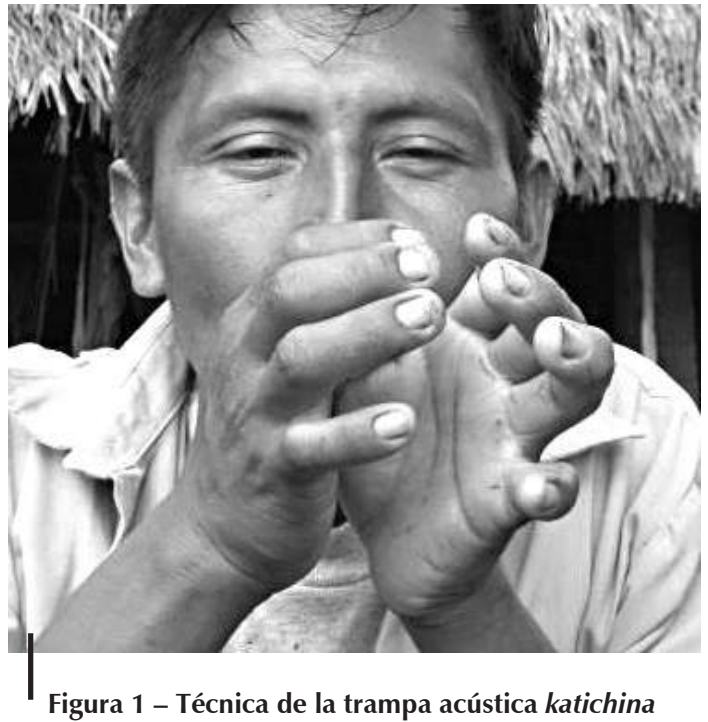

(c) Andrea-Luz Gutiérrez Choquevilca [1977]: 141), los pirahãs (Everett, 1986), los grupos gavião-mónde (Moore, 1998) y los awa-guajas (Uira Garcia, 2010; Cormier, 2003); en Perú, entre los yaguas (Chaumeil, 1993: 416-417).

Esta muda vocal se convierte en una forma de mind trap («trampa cognitiva»)10 a medida que se desempeña un diálogo entre el cazador y el animal, cuyo objetivo es literalmente producir afectos musyachi- «hacer sentir emoción» y llakichi«hacer sentir tristeza» al interlocutor no humano (Gutiérrez Choquevilca, 2013). En esta relación, el predador humano ofrece una máscara sonora «animalizada» mientras que se establece un contexto comunicacional paradójico, basado en la duplicidad enunciativa del locutor. La peculiaridad de esta forma de comunicación es que ninguna de las entidades en presencia ocupa una posición fija y estabilizada: se sugiere que al instante crítico del encuentro entre el hombre y el pájaro o el mono araña disimulado en la cubierta forestal, la reflexividad del cazador se confunde con la imagen en anamorfosis del interlocutor animal, mientras el animal deja por un breve instante de ser una presa, para verse atribuido la posición de «agente» en la interacción sonora. El cazador entra en una relación de identificación pragmática con el animal, pocos minutos antes del sacrificio, y eso por dos razones: no solo por el hecho que consigue ocupar en el marco de la relación interespecífica, un posición de «familiar», «miembro del ayllu» a veces «hermano» wawki o «cuñado» masha de su presa, según las testimonios propios de los cazadores; pero también, porque las condiciones de interacción —las facultades cognitivas involucradas, los conocimientos

10 La expresión «trampa cognitiva» proviene del análisis de los instrumentos rombos por P. Smith (1979) y ha sido examinada en detalle por A. Gell bajo la traducción mind trap acerca de las trampas de cacería exhibidos en el museo «Center for African Art» de Nueva York (Gell, 1996). 
etológicos del comportamiento animal, la espera de una respuesta, la adaptación del mensaje- proporcionan los medios para una metarrepresentación de la perspectiva no humana por el cazador. Es decir que la constitución del Otro no humano como interlocutor y agente no antecede, más bien es la consecuencia pragmática que surge del diálogo interespecífico.

Sin embargo, mas allá de la estrategia de adopción de una forma de comunicación icónica a fin de conmover profundamente y desestabilizar al interlocutor no humano, existe un repertorio de cantos rituales llamados kayachina destinados a seducir y atraer a los animales del monte mediante el establecimiento de una relación con el espíritu dueño de los animales. Empezaremos con la descripción de la epistemología propia de este tipo de performancia y seguiremos con la descripción de uno de dichos cantos kayachina. La transmisión se efectúa mediante la relación avuncular o la relación de filiación directa padre-hijo, a través de un aprendizaje formalizado, necesitando la toma de un brebaje a base de una planta alucinógena llamada marikawa (Brugmansia suaveolens, Solanaceae) en condiciones similares a los rituales arutam entre los achuars y shuars ecuatorianos (Pelizarro, 1988: 118163; Taylor, 1993). En las visiones muskuy, varios espíritus dueños de los animales genéricamente llamados sacha kuraka — se presentan bajo su apariencia zoomorfa y piden al novicio que repita con su propia voz el silbido del ave Inchichi, dueño de los jabalís generalmente identificado como un pájaro llamado wiskunchu (Lipaugus vociferans, Cotingidae), con el fin de memorizar un canto usado por el cazador o el yachak (especialista ritual, literalmente «el que sabe») para amansar y atraer a los animales. Se empleará según un protocolo específico (dieta sasina, abstinencia sexual, etc.). En otros términos, la memorización de un contenido semántico complejo se efectúa mediante la memorización de un grito y la mímesis de la voz de un pájaro, es decir una forma de comunicación icónica característica de las representaciones reflexivas del aprendizaje. Encontramos en este metacontexto de memorización, un esquema de sinonimia musical entre el acto de mímesis alucinatorio del canto de una entidad no humana (silbido de un pájaro, sonido de la rana wanip, etc.), el lenguaje articulado (el canto mismo) y una melodía tocada con la flauta traversa pupuchi confeccionada con un bambú maruna (figs. 2 y 3 ). Desde la perspectiva del cazador, se considera que estos sonidos pueden sustituirse y poseen un eficacia semejante. Asimismo, pese a la heterogeneidad de los sonidos producidos en cada contexto, el taxón utilizado para referirse a los tres tipos de performancias a través de una metonimia instrumental es único: el sintagma /kayachi-na/ (llamar-causativo-nominalizador) cuyo significado es «hacer llamar».

Sugerimos que este modo de aprendizaje no es casual. Más bien, se trata en las tradiciones orales amazónicas de un modo muy común de transmisión basado en el poder de derivación de la expresión sonora a partir de un número limitado de modelos sencillos, gritos de animales, silbidos de pájaros o motivos musicales percibidos en la experiencia onírica (Graham, 1994: 727; Gow, 2001: 147-148; Seeger, 1987: 53-56; Cesarino, 2011).

Nos parece relevante que la comunicación icónica mediante el acto de mímesis de las voces no humanas sustituye aquí al acto de nombrar a las entidades 


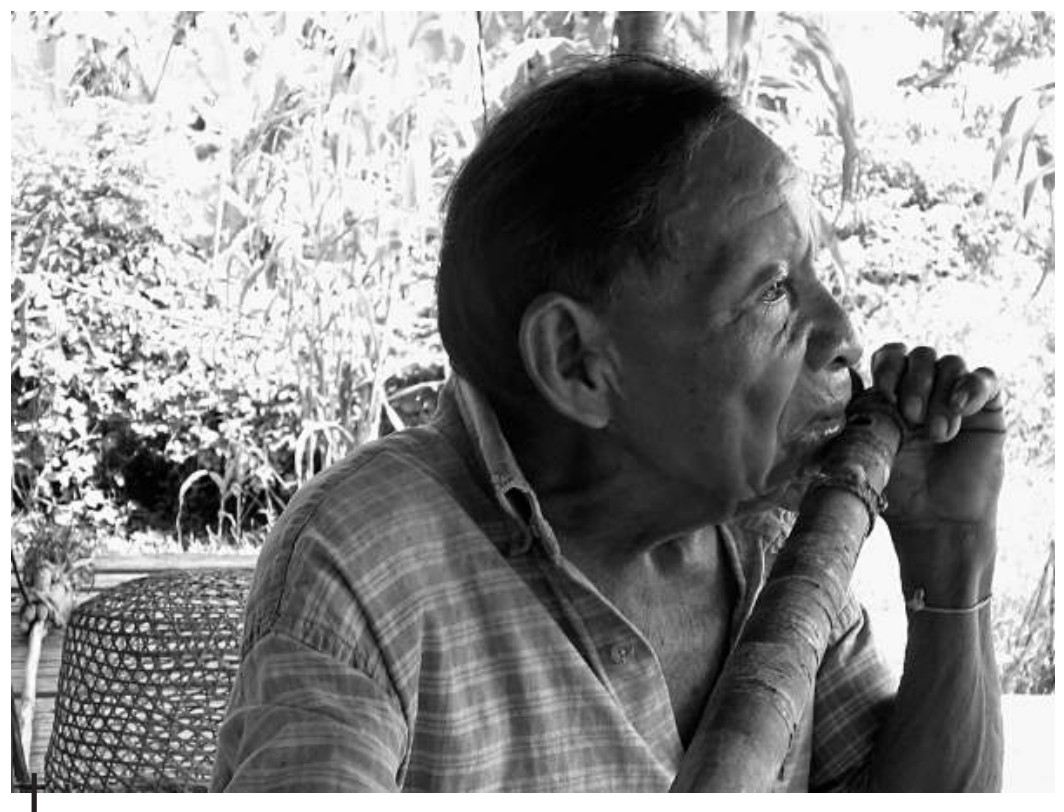

Figura 2 - Hipuli Arawanaza tocando un ikara kayachina

(C) Andrea-Luz Gutiérrez Choquevilca

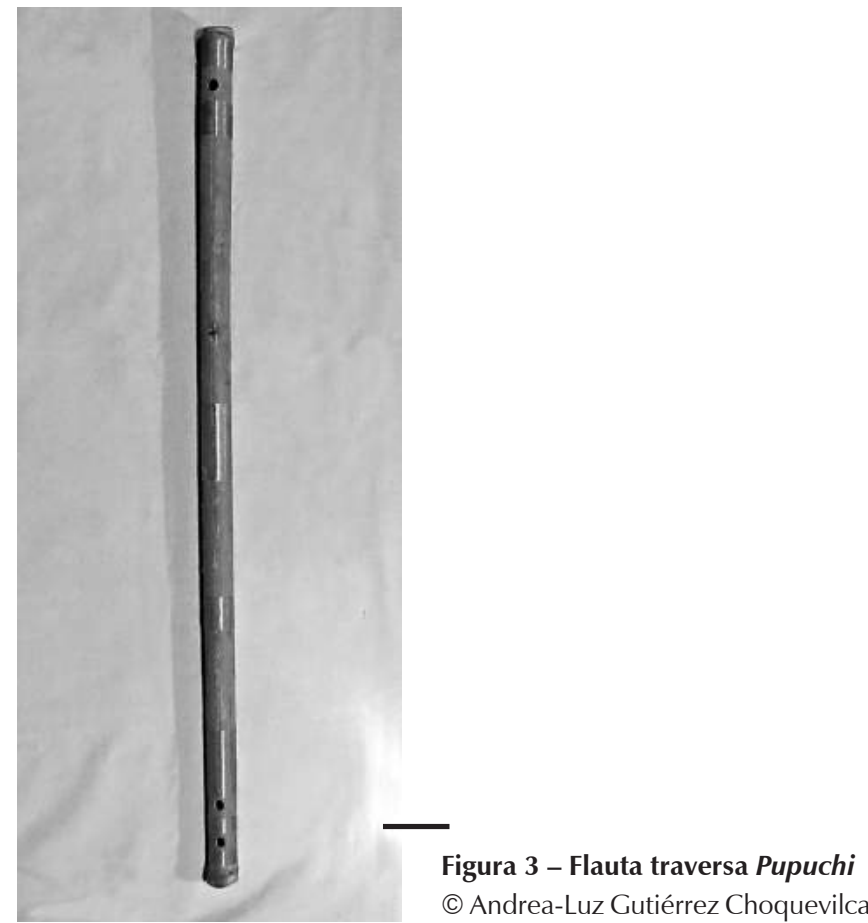


convocadas. Este conocimiento esotérico del cazador iniciado se aclara cuando tomamos en consideración a lo que se refiere Lévi-Strauss en la parte final de L'homme Nu, la «mitología implícita» del rito (1971: 598). En efecto, en el mito de Yushinku —dueño de los animales entre los runas—, se evidencia la correlación, desde la perspectiva del iniciado yachak, entre el acto onomatúrgico de nombrar a las entidades y el control adquirido sobre estas. Encontramos esa correlación entre nominación y maestría en varias otras tradiciones rituales amerindias, tales como la de los suya en Brasil, de los wakuenaï y warao del Venezuela o de los marubo (Seeger, 1987: 42-43; Hill, 1993: 22; Olsen, 1974: 93; 1980: 135-140; Cesarino, 2011: 229). Entre los runas del Pastaza, el dueño de los animales Yushinku es el depositario de un conocimiento auditivo constituido por la lista de los nombres primitivos (en su versión acústica) asignados a la voz de los animales silvestres11.

[...] «iKayta pupuchishpayki tukuy pawshi mmh $\boldsymbol{m} \boldsymbol{m} \boldsymbol{h}$ mmh wankana kurun kurun kuruun chuwa ahahaaay shutikunata yuyarinki!

Tukuy mitayu shutinkunata yuyarishpayki pupuchishina kenanki...»

Chasnashi karan chishita sirihunahunshi yachachirayanshi kampamentunkunapi kumpanyashpan:

«Kasna pupuchinki mitayu shutinkunapi kayanki!»
«iCuando toques esta flauta pupuchi, pensarás en los nombres del paujil (Crax mitu) $\mathbf{m} \boldsymbol{m} \boldsymbol{h} \mathbf{m} \boldsymbol{m} \boldsymbol{h} \boldsymbol{m} \boldsymbol{m} \boldsymbol{h}$, del jabalí (Tayassu tajacu) kurun kurun kuruun del mono chuwa ahahaaay (Ateles paniscus)!

iPensando en todos los nombres de cada uno de ellos, tocarás con tu pupuchi sus nombres!».

Así dice, al atardecer se echaba para tomar el brebaje de marikawa (Brugmansia suaveolens, Solanaceae) y el joven se echaba. Le enseñaba los nombres de los animales».

«iCon tu pupuchi toca y llama a los animales cada uno por su nombre!».

La coincidencia entre el acto de nominación musical shutiyachina y el control adquirido sobre las entidades designadas nos permite dar un nuevo enfoque sobre la noción de maestría bastante glosada en la literatura americanista, poniendo énfasis no solo en su dimensión sociológica (materializada por la relación de «filiación adoptiva» y/o pet keeping, por ejemplo) (Fausto, 2008), política o espacial (Kohn, 2007: 107-111) pero también discursiva y pragmática. La etnografía de los runas del Pastaza sugiere que el «maestro», llamado amu en el lenguaje ordinario, es el que puede ocupar un posición de «locutor especial», capaz de dominar varios códigos semióticos y de comunicar mas allá de las fronteras lingüísticas y biológicas que separan a los seres humanos y no humanos. La maestría podría coincidir aquí con el poder de nombrar o expresar mediante un tipo de comunicación icónica, un aspecto esencial de las entidades convocadas: es decir su voz, en su dimensión

11 La versión integral del mito de Yushinku, una figura prominente del dueño de los animales entre los runa del Pastaza, se encuentra analizada con mayor detallae en Gutiérrez Choquevilca (2012, vol. 2). 
primitiva no articulada. El poder de fascinación vinculado con el acto de mímesis sonora sería parte de la eficacia obtenida, como resultado de esta tensión semiótica entre la concepción indígena del lenguaje y su propia musicalidad.

\section{CITACIÓN}

La afinidad entre el proceso de comunicación icónica y el proceso de memorización merece una atención especial, porque tal esquema de transmisión del lenguaje chamánico tiene consecuencias importantes sobre la composición poética de los cantos kayachina. Como lo hemos subrayado, el proceso de citación de la voz observado durante la iniciación se refleja en la poética de los cantos mediante el uso de onomatopeyas específicas. En un canto kayachina dirigido al kuraka —señor, jefe político- de los jabalís se escucha la voz de las manadas de jabalís wankana (Tayassu tajacu) imitada por el cazador bajo la forma de un grito lexicalizado: «kurun kurun kurun». Vemos aquí el extracto del canto correspondiente, grabado con una anciana sinchi warmi, de la comunidad de Andoas, Maria Mukushua Sandi:

1 Wankanaka kurakay

wankanaka kurakay

wankanaka kurakay

shamuy shamuy shamuyaa

5 wankanaka kurakay

shamuy shamuy shamuyaa

sinchi sinchi kurnita

kurnitawami uyariii'

kayamuni kantaya

10 kayamuni kayani

kayamuni kayani

atun atun kuraka

kurakalla wankana

wankanalla kuraka

15 Shamuy shamuy wankana

Kurun kurun shamunki

Shamuy shamuy kurun kurun

Kurun kurun kurun kurun

Kurun kurun kurun kurun

20

Wankanalla wankana

Kurun kurun shamunki

kurun kurun shamunki

kurun kurun shamunki
Señor «Kuraka» del jabalí

Dueño del jabalí

Dueño del jabalí

iVen! iAcércate!

Dueño del jabalí

iVen! iAcércate!

Con el sonido fuerte de mi corneta

Con mi corneta hago sonar mi voz

Te llamo a ti mismo

Llamo y estoy Ilamando

Llamo y estoy llamando

Gran dueño kuraka

Verdadero dueño del jabalí

Dueño de la manada de jabalís

¡Ven acércate jabalí!

Kurun kurun estás viniendo

iVen! kurun kurun

kurun kurun kurun kurun

kurun kurun kurun kurun

¡Una manada de jabalís!

Kurun kurun estás viniendo

Kurun kurun estás viniendo

Kurun kurun estás viniendo 
Atun wayra yariya

25 Achwakunatalla

Urmachi' urmachi' yariy

Rurashpaylla llukshinki

Wankanalla kuraka

kurakalla kuraka

30 Atun atun wayra yariya

kurakami kantalla

Kanta yari kayamun

Atun atun yariya

Kurakami kantaya

35 Kurakami kayamun

atun atun kuraka

atun atun kuraka

Kurakami kayamun

Kurakami kayamun

40 Kwarentaykiyariya

kormitashpayni yariy'

kanta kayamuyniya

kormitashpayni yariy'

kanta kayamuyniya

45 Ririririririiiiiii

Ririririririiiiiii

Atun atun yariya

Atun atun yariya

Kormitawami yari

50 Kayamuni kayani

Kayamuni kayani

Kayamuni kantaka

Kayamuni kantaka
En el medio del aire [se siente un viento fuerte]

allí en los aguajales (Mauritia sp., Arecaceae)

[los] haces caer [y]

¡Haciendo caer las frutas de los árboles, estás saliendo!

iDueño de la manada de jabalís!

iVerdadero dueño de los dueños!

En el medio del aire [se siente un viento fuerte]

El verdadero dueño kuraka a ti mismo

Te está llamando con su voz

[al pequeño kuraka]

iSiente la inmensidad! (el viento fuerte)

El verdadero dueño kuraka a ti

El verdadero dueño kuraka te está Ilamando

iEl gran dueño kuraka!

iEl gran dueño kuraka!

El verdadero dueño kuraka está llamando

El verdadero dueño kuraka está llamando

Con tu motor fuera de borde cuarenta

haciendo escuchar el sonido de mi corneta

a ti te llamo nomás

haciendo escuchar el sonido de mi corneta

a ti te llamo nomás

\section{Ririririririiiiiii \\ Ririririririiiiiii}

iSiente la inmensidad!

iSiente la inmensidad!

Con mi verdadera corneta

Llamo y estoy llamando

Llamo y estoy llamando

iA ti te estoy llamando!

iA ti te estoy llamando! 
Washapartimantaya shamunki De atrás nomás vienes

55 Shamuy kalpa kalpa shamuyaa

Wankanalla kuraka

Wankanalla kuraka

Wankanalla kuraka

Atun yari' yariya

60 atun yari' yariya

Marchawpimantamiyari shamuy!

Shamunkiya kurakay

kayachishpayniyariya

65

Ririririririririiiiii

Ririririririririiiiiii

Ririririririririiiiiii

Ririririririririiiiiii

Ririririririririiiiiii

Ririririririririiiiiii

$70 \quad$ Ririririririririiiiiii

Ririririririririiiiiii

Wankanalla kurakay

Kurakaya kuraka!
¡Acércate corriendo! iVen corriendo!

iDueño de la manada de jabalís!

iDueño de la manada de jabalís!

iDueño de la manada de jabalís!

iSiente la inmensidad!

iSiente la inmensidad!

Desde el medio del mar ven!

¡Estás viniendo kuraka cuando te hago Ilamar!
Riririririririririiiii

Riririririririririiiiii

Riririririririririiiii

Riririririririririiiiii

Riririririririririiiii

Riririririririririiiiii

\section{Riririririririririiiii \\ Riririririririririiiii}

iDueño de la manada de jabalís!

iVerdadero dueño de los dueños!

Observamos una organización formal paralelística combinando repeticiones (a nivel sintáctico y semántico) con variables mínimas basadas en los lugares recorridos por el espíritu dueño de los animales, kuraka: el cielo y el «mar» concebido como un espacio liminal propio a los espíritus «dueños de los animales» (v. 24-25; 6162). La exhortación directa en una forma performativa —/kan-ta kaya-mu-yni-ya/: /PR2-A Ilamar-CISL-1P-ENF/ «a ti te llamo nomás»_ juega un papel clave ya que se sugiere una correlación o equivalencia pragmática entre la proposición reflexiva reiterada /kaya-mu-ni kan-ta-ka/: Ilamar-CISL-1P PR.2-A-TOP («Te llamo a ti») y el uso de una máscara sonora mediante los onomatopeyas (v. 16-23). Una presencia sonora está performada por el proceso de mímesis de la vOz, aquí refiriéndose a la voz del jabalí o del motor Johnson 40 — vehículo del dueño asimilado a la figura prominente del extranjero wirakucha. La citación de la «voz» del motor Riririririririririiiii (v. 63-70) en el canto genera una reducción de la distancia entre el evento narrado y el marco presente hic et nunc de las interacciones rituales. Sugerimos que esta epifanía sonora podría desempeñar el papel de una máscara, revelando en un paisaje auditivo (soundscape) la cara visible, sonora, de los actores 
invisibles en el marco de las interacciones rituales. El enunciador ritual vuelve a ocupar la posición ambigua de objeto y sujeto del discurso ritual, ambivalencia ya evocada en el marco de las biografías de los iniciados. Hemos sugerido en un trabajo anterior, a partir de otros datos presentando características similares (el uso de la comunicación icónica, de la citación directa o indirecta de las voces invisibles), que el carácter performativo de los cantos kayachina resulta no tanto del contenido semántico referencial del discurso, sino de la manipulación sonora del contexto de interacción, mediante el mecanismo central de citación - la usurpación de la voz de los espíritus dueños de los animales sacha kuraka (Gutiérrez Choquevilca, 2011)_. La autoridad del yachak reside en el poder de intercambiar su voz, de exponer una máscara sonora simultáneamente humana y no humana en la sincronía de la performancia ritual. Si bien se ha demostrado en una amplia literatura americanista la incidencia del concepto de metamorfosis — cambio de «piel» en correlación con un cambio de perspectiva12_ en el aprendizaje del pensamiento animista y en su transposición en el campo ritual, sugerimos aquí que el concepto de transformación o metamorfosis, encontraría en este contexto un campo de aplicación más allá de las categorías morfológicas y visuales, aquí expresado en la dimensión acústica: una metamorfosis de la voz (Gutiérrez Choquevilca, 2012; Cesarino, 2011). En este sentido, el uso de la citación, de igual manera como el uso de las onomatopeyas o de un modo de comunicación icónico, podrían desempeñar un papel clave permitiendo definir nuevamente en el marco de las interacciones rituales, la identidad de los participantes (véase Hanks, 2009; para un análisis complementario del mismo proceso en el marco del chamanismo maya) ${ }^{13}$. Si bien la relación íntima entre el uso del sonido y la transformación de las relaciones establecidas en el ritual aparece claramente en el contexto de las actividades de cacería, cabe señalar otro repertorio presente en la tradición ritual de los runas amazónicos, donde el uso del sonido y del proceso de citación de una voz no humana desempeña un papel preformativo. Se trata de los cantos líricos o cantos de amor conocidos como llakichina (tratándose de la nominalización de la raíz verbal llaki- denotando el sentimiento ambiguo de amor y tristeza bajo la forma causativa: «hacer sentir tristeza»). Una categoría musical similar existe en la tradición achuar bajo el taxón «anent» (entre otros Taylos \& Chau, 1983). La mayoría de los cantos cayendo bajo la categoría llakichina del repertorio tienen como característica referirse a la relación de pareja como a una relación de complicidad intraespecífica entre dos pájaros hembra y macho de la misma especie ocupando un ecosistema divido entre tierras altas y tierras bajas: según los intérpretes, se trataría principalmente del tucán sikwanka (Ramphastos sp., Ramphastidae), del pájaro kamunkuy (Anhima cornuta, Anhimidae) o del pájaro wishuinshu (Lipaugus vociferans, Cotingidae) cuya voz es similar al sonido de una flauta de émbolo.

Cabe recordar que el modo de aprendizaje de estos cantos es semejante al contexto mencionado anteriormente de iniciación cinegética, ya que se trata de reproducir

\footnotetext{
12 Véase Viveiros de Castro, 1986; Seeger, 1975; entre otros.

13 Veanse Rumsey, 2000; Urban, 1989, para un estudio de la flexibilidad referencial de los marcadores indexicales de la primera persona, provocando un efecto similar.
} 
o imitar en un metacontexto de visiones inducidas por la toma de sustancias psicotrópicas marikawa (Brugmansia suaevolens, Solanaceae), la voz de un pájaro o de una rana wanipa con el fin de memorizar el contenido articulado de los cantos. Este género musical viene a provocar emociones muy fuertes en el destinatario de las palabras rituales, que suelen ser enunciadas en la soledad y en voz baja. A menudo se describe el efecto conativo de los cantos como la irrupción de una profunda tristeza y melancolía enlazando al locutor y al destinatario ausente de la performancia. La enunciación de los cantos ocurre generalmente al anochecer o al amanecer, y es seguida de la toma de jugo de tabaco ohampa (Nicotiana sp.) destinada a desenvolver la concentración y aclarar las visiones del enunciador ritual. La asimilación del poder de los nombres o sonónimos enunciados con la eficacia de las flechas mágicas wiruti características del modo de acción terapéutico, es patente.

1 Kamunkuynistuya uyarin!

Karumanta kawn 'kuy!

Urkumanta kawn 'kuy! kawn 'kuy!

Uyari'lla kankaya

5 kanka yariy kantaka!

Upyaysikistuyniya

parihumi ñukanchika!

Maypichari yariyaa!

yakutalla tarinchi

10 chaypimiya upyashu!

Kamunkuynistu uyarin

Karumanta ka'ùun'mkuy!

Warmimiya kankaya

ñukakaya kantaya!

15 Kamunkuynistuya uyarin!

Karumanta kawn 'kuy!

Urkumanta kawn 'kuy! kawn 'kuy!

Uyari'lla kankaya

Kayllayashkani yariy

20 mana sakishkaykichu!

Kamunkuynistu uyarin kawn 'kuy!

kawn 'kuy!
¡La voz de mi pequeño pájaro kawn'kuy' suena!

iDesde lejos suena kawn'kuy'!

iDesde la altura suena kawn 'kuy! kawn 'kuy!

Tu voz no deja de sonar

iTú mismo, siente!

Mi pequeño tomalón (el que sabe tragar)

Asimismo verdaderamente nosotros!

iDonde estés escucha!

iEncontramos agua pura,

en este árbol, tomemos!

La voz de mi pájaro kamun'kuy' suena

iDesde lejos, ka'ùun'mkuy!

Tú mismo eres una mujer

iYo mismo (me dirijo) a ti!

iLa voz de mi pequeño pájaro suena kawn'kuy'!

iDesde lejos suena kawn'kuy'!

iDesde la altura suena kawn 'kuy! kawn'kuy!

Tu voz no deja de sonar

iMe estoy acercando, siente!

No te puedo abandonar

iLa voz suena kawn 'kuy! kawn 'kuyi 
Respecto a las características musicales del canto llakichina, observamos una alternancia entre dos registros musicales con una voz alta en falcetto (A) y una voz baja (B), con variaciones $\left(\mathrm{A}^{\prime}-\mathrm{B}^{\prime}\right)$ encajada en una estructura musical repetitiva.

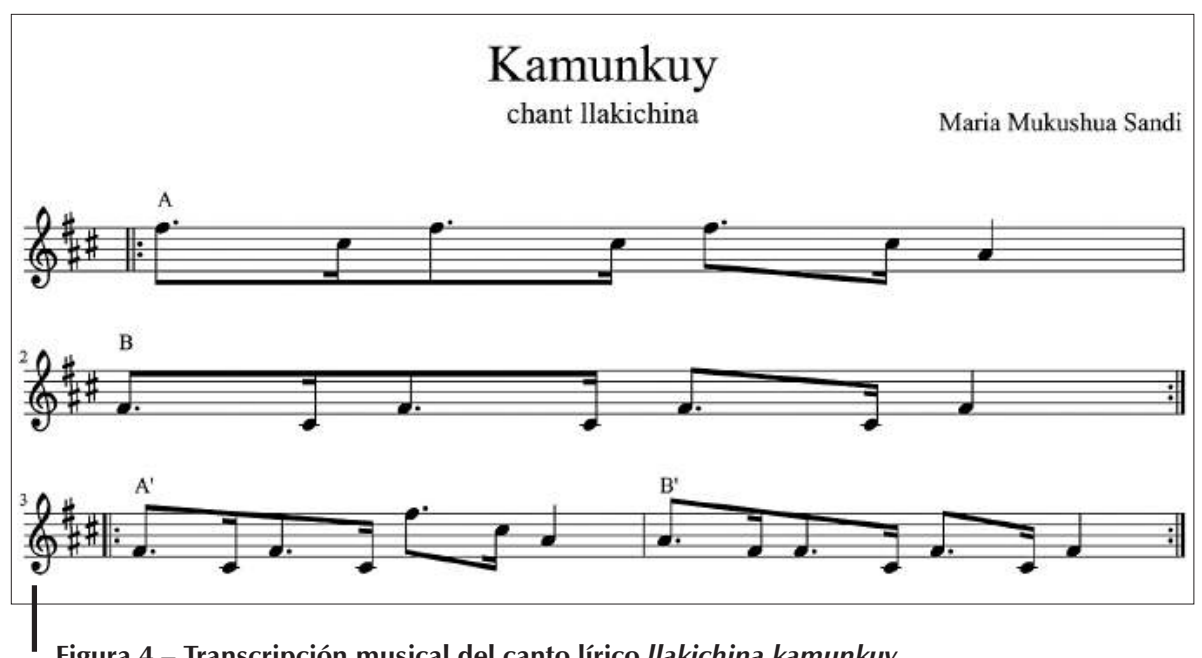

Figura 4 - Transcripción musical del canto lírico llakichina kamunkuy

Se evidencia que el uso de las onomatopeyas «kawn 'kuy! kawn 'kuy!», a fin de nombrar a las entidades convocadas, no solo permite citar la voz del enunciador lírico, mascada por el canto de un pájaro, sino que establece un cambio de marco interaccional. La relación afinal entre los participantes se superpone a una relación de complementariedad ecológica entre los pájaros de las tierras bajas y de altura. Nuevamente se destaca a través del uso de la citación o de la comunicación icónica — una metalengua no humana - una nueva definición de las identidades rituales de los participantes. Contribuye a crear un campo de interacción específico donde estas identidades, la del enunciador lírico y la de las entidades no humanas se vuelven redistribuidas o sustituidas. Otra técnica retórica común es la concatenación de las marcas deícticas que alternan, a fin de integrar en una cadena poética, la copresencia del enunciador ideal (pájaro kamunkuy identificado como una mujer, en un posición femenina) y del destinatario (pájaro-cónyuge). En los versos siguientes, se ve condensada a través de la sucesión de los pronombres de la primera y segunda persona:

$\begin{array}{lll}\text { v. } 13 & \text { Warmi-mi-ya } & \text { kan-ka-ya } \\ & \text { Mujer-ASS-ENF } & \text { PR2-TOP-ENF }\end{array}$

Tú mismo eres una mujer

v. 14 ñukakaya kantaya

PR1-TOP-ENF PR2-ACC-ENF

iYo mismo (me dirijo) a ti! 
Sugerimos para concluir, que una antropología pragmática del sonido y de la voz en el marco del discurso ritual podría permitir dar un nuevo enfoque sobre la noción de metamorfosis, que aparece en la etnografía como una característica fundamental de las ontologías anímicas. Mi hipótesis es que el uso de la voz podría funcionar en el marco de las interacciones entre humanos y no humanos (animales o espíritus) como un instrumento de transformación central de la identidad de los actores rituales. A través del estudio pragmático de los cantos rituales quechuas, hemos podido diferenciar varios modelos de interacción: el primero se evidencia en el contexto de enunciación de los cantos kayachina, coincidiendo con el uso de una «máscara acústica»: el enunciador usurpa la posición del «espíritu-dueño» de los animales. El segundo tipo evocado en los cantos de amor Ilakichina se refiere a una manipulación simbólica de las relaciones interindividuales a través de la confrontación dialógica de los participantes con interlocutores no humanos representados en los cantos mediante la imitación de las voces de unos pájaros. El análisis sugiere que las técnicas de comunicación acústicas observadas en el lenguaje ritual quechua juegan un papel clave no solo en el marco de las interacciones entre humanos y no humanos en el contexto de las expediciones de cacería, sino también en la eficacia del proceso de comunicación ritual donde, a través de las máscaras acústicas, los actores visibles se vuelven invisibles y los invisibles se vuelven audibles.

\section{Referencias citadas}

BASSO, E. B., 1985 - A musical view of the universe: kalapalo myth and ritual performances, xvi +343 pp.; Philadelphia: University of Pennsylvania Press.

BEAUDET, J. M., 1997 [1977] - Souffles d’ Amazonie : les orchestres «Tule» des Wayãpi, 212 pp.; Nanterre: Société d'Ethnologie.

CESARINO, P. N., 2011 - Entre la parole et l'image. Le système mythopoétique Marubo. Journal de la Société des Américanistes, 97 (1): 223-257.

CHAUMEIL, J.-P., 1993 - Des esprits aux ancêtres. Procédés linguistiques, conception du langage et de la société chez les Yagua de l'Amazonie péruvienne. L'Homme, 33 (126-128): 409-427.

CORMIER, L. A., 2003 - Kinship with Monkeys: The Guajá Foragers of Eastern Amazonia, xxvi + 234 pp.; Nueva York: Columbia University Press.

DÉLÉAGE, P., 2009 - Le chant de I'anaconda. L'apprentissage du chamanisme chez les Sharanahua (Amazonie occidentale), 409 pp.; Nanterre: Société d'ethnologie.

EVERETT, D. L., 1986 - Pirahã. In: Handbook of Amazonian Languages (D. Derbyshire \& G. Pullum, eds.), vol. I: 200-226; Berlín, Nueva York: Mouton de Gruyter.

FAUSTO, C., 2008 - Donos demais: maestria e domínio a Amazônia. Mana, 14 (2): 329366.

FAUSTO, C., 2011 - Le masque de l'animiste. Chimères et poupées russes en Amérique indigène. Gradhiva, Pièges à voir, pièges à penser, 13: 49-67. 
FAUSTO, C., FRANCHETTO, B. \& MONTAGNANI, T., 2011 - Les formes de la mémoire : Art verbal et musique chez les Kuikuru du haut-Xingu (Brésil). L’homme, 197 (1): 41-70.

GELL, A., 1996 - Vogel's Net: Traps as Artworks and Artworks as Traps. Journal of Material Culture, 1 (1): 15-38.

GOULARD, J.-P. \& KARADIMAS, D. (eds.), 2011 - Masques des hommes, visages des dieux, 311 pp.; París: Centre national de la recherche scientifique.

GOW, P., 2001 - An Amazonian myth and its history, xiii + 338 pp.; Oxford: Oxford University Press.

GRAHAM, L., 1994 - Dialogic dreams: creatives selves coming into life in the flow of time. American Ethnologist, 21 (4): 723-745.

GUTIÉRREZ CHOQUEVILCA, A.-L., 2011 - Sisyawaytii tarawaytii. Sifflements serpentins et autres voix d'esprits dans le chamanisme quechua du haut Pastaza (Amazonie péruvienne). Journal de la Société des Américanistes, 97 (1): 179-222.

GUTIÉRREZ CHOQUEVILCA, A.-L., 2012 - Voix de maitres et chants d'oiseaux. Pour une étude pragmatique de l'univers sonore et de la communication rituelle parmi les peuples Quechua d'Amazonie péruvienne, 2 vols., 1115 pp.; Nanterre: Université Paris X. Tesis de doctorado.

GUTIÉRREZ CHOQUEVILCA, A.-L., 2013 - Face-à-face interspécifiques et pièges à pensée des Quechua de Haute Amazonie. Cahiers d'Anthropologie Sociale, n. ${ }^{\circ}$ 9: 33-47; París: L'Herne Éditions, Collège de France.

HANKS, W. F., 2009 - Comment établir un terrain d'entente dans un rituel ? In: Paroles en actes (C. Severi \& J. Bonhomme, eds.), vol. 5: 87-115; París: L'Herne.

HILL, J. D., 1993 - Keepers of the Sacred Chants: the poetics of ritual in an Amazonian society, xix + 245 pp.; Tucson, London: The University of Arizona Press.

HILL, J. D. \& CHAUMEIL, J.-P. (eds.), 2011 - Burst of Breath Indigenous Ritual Wind Instruments in Lowland South America, 440 pp.; Lincoln: University of Neebraska Press.

HOUSEMAN, M., 2008 - Relations rituelles et recontextualisation. In: Multiplicity of Meaning and the Interrelationship of the Subject and the Object in Ritual and Body Texts (H. Wazaki, ed.): 109-114; Nagoya: Nagoya University.

KOHN O., E., 2007 - Animal Masters and the Ecological Embedding of History among the Avila Runa of Amazonian Ecuador. In: When Time Matters: History, Memory, and Identity in Amazonia (C. Fausto \& M. Heckengerger, eds.): 106-129; Florida: University of Florida Press.

LÉVI-STRAUSS, C., 1949 - L'efficacité symbolique. Revue de l'Histoire des Religions, 135 (1): 5-27

LÉVI-STRAUSS, C., 1971 - L'homme nu, 688 pp.; París: Plon.

MELLO, M. I. CRUZ, 2005 - lamurikuma: música, mito e ritual entre os Wauja do Alto Xingu, 335 pp.; Florianópolis: Universidade Federal de Santa Catarina. Tesis de doctorado en Antropología Social.

MENEZES BASTOS, R. J. de., 1990 - A festa da jaguatirica: uma partitura críticointerpretativa, xvi +607 pp.; São Paulo: Universidade de São Paulo. Tesis de doctorado en Antropología.

MICHAEL, L., BEIER, C., ESCAMILLA, R. \& PIQUERAS BRUNET, M., 2009 - Katsakáti: El idioma antiguo del pueblo de Andoas. Ms.

MONTAGNANI, T., 2011 - Présences sonores. Musique, images et langue chez les Kuikuro du Haut Xingu. Images Re-vues, 8: 1-13. Disponible en http://imagesrevues.revues. org/493. 
MOORE, D., 1998 - Tonal system of the Gavião Language of Rondönia, Brazil, in Tupian Perspective. In: Proceedings of the Symposium "Cross-Linguistic Studies of Tonal Phenomena, Tonogenesis, Typology, and Related Topics" (S. Kaji, ed.): 297-310; Tokyo: Institute for the Study of Languages and Cultures of Asis and Africa, Tokyo Institute of Foreign Studies.

MURATORIO, B., 1987 - Rucuyaya Alonso y la historia social y económica del Alto Napo 1850-1950, 341 pp.; Quito: Ediciones Abya Yala.

OLSEN, D. A., 1974 - The functions of naming in the curing songs of the Warao Indians of Venezuela. Annuario Interamericano de Investigación musical, 10: 88-122.

OLSEN, D. A., 1980 - Magical protection songs of the Warao Indians. Latin American Music Review, 1 (2): 131-161.

OLSEN, D. A., 1996 - Music of the Warao of Venezuela: song people of the Rain Forest, xxxiv + 444 pp.; Gainesville: University Press of Florida.

OVERING, J., 1990 - The shaman as a maker of worlds: Nelson Goodman in the Amazon. Man, 25 (4): 602-620.

PEEKE, C., 1954 - Shimigae: idioma que se extingue. Perú Indígena, 5: 171-178.

PEEKE, C. \& SARGENT, M., 1953 - Pronombres personales en andoa. Perú Indígena, 5: 103-112.

PELIZZARO, S. M. (ed.), 1988 - Arutam. Mitos de los espíritus y ritos para propiciarlos, 195 pp.; Quito: Abya Yala.

PIEDADE, A. T. de CAMARGO, 1997 - Música yepamasa: por uma antropologia da música no Alto Rio Negro; Florianópolis: Universidade Federal de Santa Catarina. Tesis de maestría en Antropología Social.

REEVE, M.-E., 1988 - Cauchu Uras: Lowland Quichua Histories of the Amazon Rubber Boom. In: Rethinking History and Myth: Indigenous South American Perspectives on the Past (J. D. Hill, ed.): 21-34; Urbana: University of Illinois Press.

REEVE, M.-E., 1993-1994 - Narratives of a catastrophe: the zaparoan experience in Amazonian Ecuador. Bulletin de la société suisse des américanistes, 57-58: 17-24.

RUMSEY, A., 2000 - Agency, personhood and the 'I' of discourse in the Pacific and beyond. Journal of the Royal Anthropological institute, 6: 101-115.

SEEGER, A., 1975 - The meaning of body ornaments: A Suya example. Ethnology, 14 (3): 211-224.

SEEGER, A., 1981 - Nature and Society in Central Brazil: The Suyá Indians of Mato Grosso, 278 pp.; Cambridge: Harvard University Press.

SEEGER, A., 1987 - Why Suyá sing: a musical anthropology of an amazonian people, xxi + 147 pp.; Cambridge: Cambridge University Press.

SEVERI, C., 2007 - Le principe de la chimère. Une anthropologie de la mémoire, 370 pp.; París: Presses de l'École Normale Supérieure (Rue d'Ulm), Musée du Quai Branly.

SMITH, P., 1979 - Aspects de l'organisation des rites. In: La fonction symbolique (M. Izard \& P. Smith, eds.): 139-170; París: Gallimard.

TAYLOR, A.-C., 1993 - Des fantômes stupéfiants. Langage et croyance dans la pensée Achuar. L'Homme, n. ${ }^{\circ}$ 126-128: 429-447.

TAYLOR, A.-C., 2007 - Sick of history: Contrasting regimes of historicity in the Upper Amazon. In: Time and Memory in Indigenous Amazonia (C. Fausto \& M. Heckenberger, eds.): 133-168; Gainesville: University Press of Florida.

TAYLOR, A.-C. \& CHAU, E., 1983 - Jivaroan magical songs: Achuar anent of connubial love. Amerindia, 8: 87-127. 
Máscaras sonoras y metamorfosis en el lenguaje ritual de los runas del Alto Pastaza (Amazonía, Perú)

TOWNSLEY, G., 1993 - Song Paths: The ways and means of Yaminahua shamanic knowledge. L'Homme, 33 (126-128): 449-468.

UIRA GARCIA, F., 2010 - Karawara. A caça e o mondo do Awá Guajá, 456 pp.; São Paulo: Universidad de São Paulo. Tesis de doctorado en Antropología Social.

URBAN, G., 1989 - The I of discourse. In: Semiotics, Self, and Society (B. Lee \& G. Urban, eds.): 26-51; Berlín, Nueva York: Mouton de Gruyter.

VIVEIROS DE CASTRO, E., 1986 - Araweté: os deuses canibais, 744 pp.; Río de Janeiro: Jorge Zahar Editor, Associação Nacional de Pós Graduação e Pesquisa em Ciências Sociais.

WHITTEN, N. E., 1976 - Sacha Runa: ethnicity and adaptation of Ecuadorian jungle Quichua, xviii + 348 pp.; Urbana: University of Illinois Press. 